\title{
EVALUACIÓN DE LA EFICIENCIA DE UN NEUTRALIZADOR DE GLICOL E INHIBIDOR DE CORROSIÓN PARA ACERO AL CARBONO EN SISTEMA DE GLICOL
}

\author{
Esteban Castellanos $^{1 *}$, Isabel Díaz-Tang ${ }^{2}$
}

\begin{abstract}
RESUMEN
Se realizó una caracterización fisicoquímica a muestras de trietilenglicol rico (en contacto con gas natural y humedad), trietilenglicol pobre TEGP (sin humedad) y agua de condensación tomadas del sistema de trietilenglicol ACT de una unidad criogénica. Se realizó pruebas de resistencia a la polarización lineal (LPR) y curvas de polarización Tafel para determinar la velocidad de corrosión del TEGR, TEGP Y ACT y la eficiencia del inhibidor de corrosión (Coastal 1755) en diferentes concentraciones para condiciones estáticas. Para la evaluación se utilizó un electrodo de anillo rotatorio acoplado a un potenciostato galvanostato Autolab/ PGSTAT 302N, utilizando procedimientos de acuerdo con la norma ASTM G-170- 01, "Standard Guide for Evaluating and Qualifying Oil Field and Refinery Corrosion Inhibitors in the Laboratory".

Se caracterizó el depósito de corrosión extraído del sistema de drenaje mediante espectrometría de emisión Jarell Ash y difracción de rayos X, marca Phillips.

La velocidad de corrosión del agua de proceso sin inyección de inhibidor está en el orden de 4 mpy $(0,114 \mathrm{~mm} / \mathrm{año})$ y para las mezclas de trietilenglicol se consideran despreciables, por lo tanto el inhibidor Coastal 1755-C está neutralizando la acidez y controlando la corrosión en el sistema.
\end{abstract}

Palabras clave: Neutralizador de glicol, inhibidor de corrosión, trietilenglicol, azufre.

\section{EVALUATING A GLYCOL NEUTRALIZER AND CORROSION INHIBITOR EFFICIENCY FOR CARBON STEEL IN A GLYCOL SYSTEM}

\begin{abstract}
A physicochemical characterization was conducted for samples of rich triethylenglycol RTEG (contacted with natural gas and moisture), poor glycol PTEG (without moisture) and water of condensation taken from a cryogenic unit TWC. Tests of linear polarization resistance (LPR) and Tafel polarization curves were performed to determine the corrosion rate of RTEG, PTEG and TWR (Teg water removed) and the corrosion inhibitor (Coastal 1755) efficiency at different concentrations for static conditions. One rotating ring electrode coupled to a potentiostat galvanostat Autolab/PGSTAT 302N was used for evaluation, using procedures in accordance with ASTM G-170- 01, "Standard Guide for Evaluating and Qualifying Oil Field and Refinery Corrosion Inhibitors in the Laboratory ".

The corrosion deposit extracted from the drainage system was characterized by an emission spectrometer, brand Jarrel Ash and x-ray diffraction brand Phillips.
\end{abstract}

1*Planta de Gas de Malvinas. Pluspetrol. Echarate, La Convención, Cuzco, Perú. E-mail: ecastell@pluspetrol.net 2 Instituto de Corrosión y Protección. Pontificia Universidad Católica del Perú, Av.Universitaria 1801, Lima 32, Perú. 
Corrosion rate of process water without inhibitor injection is in the order of $4 \mathrm{mpy}(0.114 \mathrm{~mm}$ / year) and for mixtures of triethylenglycol are considered negligible therefore Coastal 1755$\mathrm{C}$ inhibitor is neutralizing acidity and controlling corrosion in the system.

Keywords: Glycol neutralizer, corrosion inhibitor, triethylenglycol, sulfur.

\section{INTRODUCCIÓN}

En el Perú se ha establecido las siguientes plantas de procesamiento de gas natural ${ }^{1}$ :

a. Aguaytía Energy del Perú S.R.L. - Planta de Procesamiento y Fraccionamiento de Gas Natural.

b. Graña y Montero Petrolera - Planta de Gas Natural Verdún y Pariñas. (Piura).

c. Pluspetrol Perú Corporation S.A. - Planta de Separación de Gas Natural, Las Malvinas y Planta de Fraccionamiento de Líquidos de Gas Natural, Pisco.

d. Procesadora de Gas Pariñas S.A.C. - Planta Criogénica de Gas Natural.

En la unidad criogénica la corriente de gas es deshidratada hasta los valores de normativas internacionales, cercanas a $4 \mathrm{lb} / \mathrm{MMSCF}$ (libras por millón de pies cúbicos estándar por día), mediante el proceso de deshidratación con trietilenglicol (TEG) y con tamices moleculares. La unidad incluye el sistema de separación y filtración del gas a la entrada, un sistema de deshidratación que está constituido por la deshidratación con TEG que elimina una gran parte del vapor de agua, acompañado por un sistema de regeneración y deshidratación final mediante tamices moleculares. La unidad tiene un sistema de recuperación de líquidos condensables (NGL) y un sistema de compresión de gas seco.

Antes de la unidad criogénica se puede usar filtros de carbón activado impregnado de sulfuro lo que puede hacer disminuir el $\mathrm{pH}$ en el sistema de glicol, dependiendo de las condiciones de operación, lo que puede incrementar el riesgo de corrosión interna en equipos y líneas. Aparte de evidenciar la caída de $\mathrm{pH}(3,90-4,00)$ se detectan compuestos sulfurados en el gas después del paso por el tamiz. Debido a la caída de $\mathrm{pH}$ se procedió a la adición de un neutralizanteinhibidor que promoviera el incremento del $\mathrm{pH}$ hasta un valor de 7,16, aproximadamente, con la finalidad de restablecer el equilibrio al sistema.

\section{PARTE EXPERIMENTAL}

\section{Caracterización fisicoquímica de las muestras de ACT; TEGP y TEGR:}

- Mediciones de $\mathrm{pH}$ y de conductividad eléctrica usando un equipo pH-Meter marca Hach 330i para $\mathrm{pH}$ y un equipo Hach Sension 5 para conductividad.

- Determinación de: Cloruros (ppm), hierro (ppm), sulfatos, calcio y bario fueron realizados por métodos de colorimetría, titulación y espectrofotometría (espectrofotómeto Hach DR 2500).

- Determinación de sólidos disueltos (TSD) se realizó por el método de gravimetría.

- Determinación de H2S (sulfuro de hidrógeno) se llevó a cabo utilizando un kit colorimétrico marca CHEMETRICS.

\section{Evaluación electroquímica del inhibidor ${ }^{2}$ :}

- Se realizó pruebas de resistencia a la polarización lineal (LPR) y curvas de polarización de Tafel para determinar la velocidad de corrosión del GN, TEGP, TEGR y ART y la eficiencia del inhibidor de corrosión (Coastal 1755) en diferentes concentraciones, para 
condiciones estáticas. Para la evaluación se utilizó un electrodo de anillo rotatorio acoplado a un potenciostato galvanostato Autolab/PGSTAT 302N, utilizando procedimientos de acuerdo con la norma ASTM G-170-0133.

Caracterización de una muestra de productos de corrosión:

- Se caracterizó una muestra de los depósitos de corrosión extraída del sistema de drenaje de la unidad criogénica mediante espectrometría de emisión (equipo Jarell Ash) y difracción de rayos X (equipo marca Phillips).

\section{RESULTADOS Y DISCUSIÓN}

Caracterización fisicoquímica del agua condensada (ACT).

En la tabla 1 se muestran los resultados obtenidos de la evaluación fisicoquímica del ACT.

Tabla 1. Resultados de los análisis del agua condensada del TEG.

\begin{tabular}{|c|c|c|c|c|c|c|c|}
\hline Dureza total ${ }^{*} \mathrm{Ca}$ & Cloruros * & Hierro * & Alcalinidad & Sulfatos * & * Sólidos & & Conductividad \\
\hline $\mathrm{CO}_{3}$ & $\mathrm{Cl}^{-}$ & $\mathrm{Fe}$ & ${ }^{*} \mathrm{CaCO}{ }_{3}$ & $\mathrm{SO}_{4}^{-2}$ & disueltos * & $\mathrm{pH}$ & $\mu \mathrm{S} / \mathrm{cm}$ \\
\hline 14,6 & 35,0 & 2,80 & 20,0 & & 07,03 & 6,95 & 13,1 \\
\hline
\end{tabular}

$* \mathrm{mg} / \mathrm{L}$

Los valores reportados de conductividad de 13,1 micro-siemens por centímetro y el contenido de sólidos disueltos de 7,03 ppm corresponden a valores aceptables para el agua removida ya que a partir de $500 \mathrm{ppm}$ de sólidos disueltos se considera un agua ligeramente corrosiva. El contenido de hierro reportado de 2,80 ppm confirma que el agua presenta bajo nivel de corrosividad para los aceros al carbono del proceso.

Los análisis para determinar ácido sulfhídrico $\left(\mathrm{H}_{2} \mathrm{~S}\right)$ en el agua no mostraron presencia de este compuesto.

Caracterización de depósitos adheridos al tubo de drenaje del agua de proceso.
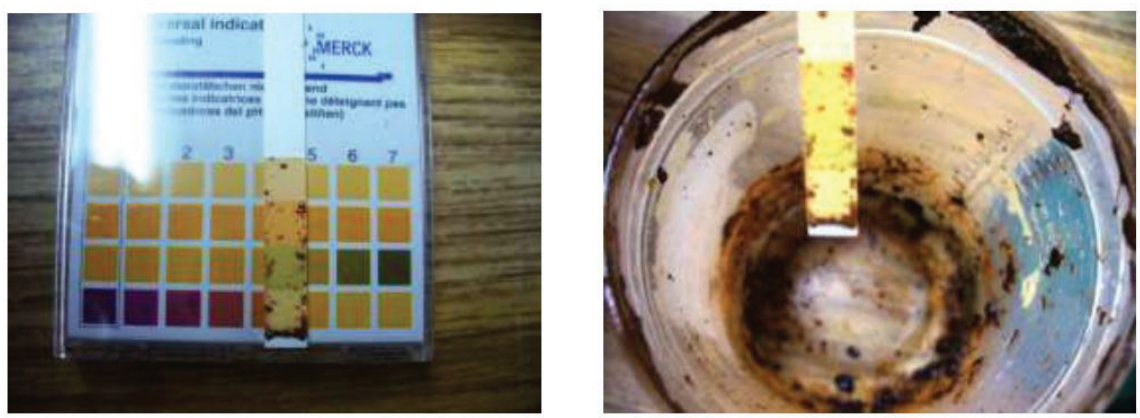

Figura 1. Medición de pH por el método del papel en la muestra extraída del drenaje de agua. El valor estimado del $\mathrm{pH}$ es de 5 . 
La muestra de depósito adherido al tubo de drenaje se presentó de forma pastosa por lo que se procedió a medir el $\mathrm{pH}$, el cual presentó un valor de $\mathrm{pH} 5$, lo que indica la presencia de compuestos ácidos en el sistema de drenaje.

A continuación se presenta los valores promedio de los elementos identificados por la técnica de espectrometría de emisión en la muestra extraída:

Tabla 2. Promedio de los elementos encontrados en el depósito extraído del drenaje

\begin{tabular}{ll}
\hline Elemento (\% peso) & Promedio \\
Oxígeno & 32,1 \\
Fierro & 40,1 \\
Azufre & 27.8 \\
\hline
\end{tabular}

Los resultados muestran un contenido de azufre del $28 \%$ en peso además de la presencia de hierro y oxígeno.

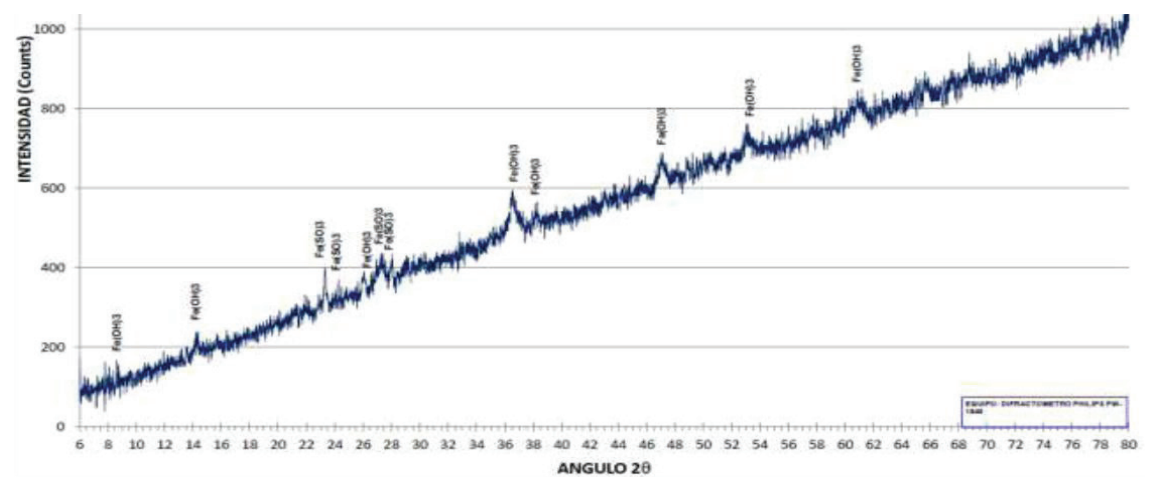

Figura 2. Espectro de difracción por rayos $X$ de la muestra de depósito removida del drenaje del agua.

En la figura 2 se presenta el análisis del depósito removido del drenaje mediante la técnica de difracción de rayos X; la cual detecta los compuestos cristalinos presentes en la muestra. Los resultados mostraron la presencia mayoritaria de sulfato de hierro hidratado e hidróxido de hierro como especies cristalinas presentes en la muestra.

\section{Caracterización del trietilenglicol pobre (TEGP) y rico (TEGR):}

A continuación, en la tabla 3 , se presenta los resultados obtenidos de la evaluación fisicoquímica del TEG pobre y el TEG rico. 
Tabla 3. Resultados de la caracterización fisicoquímica del TEG rico y pobre.

\begin{tabular}{|c|c|c|c|c|c|}
\hline & $\begin{array}{l}\text { Hierro } \\
(\mathrm{ppm})\end{array}$ & $\mathrm{pH}$ & Agua (\%) & $\begin{array}{l}\text { Sólidos } \\
\text { disueltos } \\
\mathrm{mg} / \mathrm{L}\end{array}$ & $\begin{array}{c}\text { Tendencia a } \\
\text { formar } \\
{\text { Cloruros }\left(\mid \text { espuma }^{1}\right.}^{1} \\
\end{array}$ \\
\hline TEG rico $^{2}$ & 1,70 & 8,58 & 5,10 & 0,860 & $750 \mathrm{a}=0, \mathrm{t}=0$ \\
\hline TEG pobre $^{3}$ & 0,27 & 8,54 & 2,07 & 0,720 & $575 \mathrm{a}=0, \mathrm{t}=0$ \\
\hline Especificaciones & $<15$ & $6,5-7,5$ & TEG $p<1,5$ & $<200$ & $\begin{array}{l}\mathrm{a}<300 \mathrm{ml}, \\
\mathrm{t}<15 \mathrm{~s}\end{array}$ \\
\hline
\end{tabular}

${ }^{1} \mathrm{a}=$ altura de espuma, $\mathrm{t}=$ tiempo de ruptura de la espuma.

${ }^{2}$ TEG rico $=$ trietilenglicol con agua absorbida.

${ }^{3}$ TEG pobre $=$ trietilenglicol sin agua.

Los resultados obtenidos de las muestras evaluadas de TEG rico y pobre indican lo siguiente:

- $\quad \mathrm{pH}$ : Los valores encontrados de $\mathrm{pH}(8,58$ y 8,54$)$ indican que puede haber una tendencia a la formación de espuma y de emulsiones estables en el sistema del TEG rico y pobre. Este valor puede ser atribuido al uso del inhibidor en base aminas en exceso. El valor ideal de pH para este tipo de mezclas es de 6,5-7,5.

- Cloruros: El contenido de iones de cloruro para el TEG rico está por encima del límite permitido, aumentando la probabilidad de deposición de sales en los tubos del Reboiler, causando la reducción de la eficiencia térmica y originando posibles puntos calientes en el sistema.

- Agua: El contenido de agua en el TEG pobre es superior a lo especificado en la literatura, lo cual puede indicar que el proceso de regeneración no está siendo eficiente. Sin embargo, se obtiene la relación de agua entre el TEG rico y pobre entre el 2-5 \%.

- Hierro: Los valores obtenidos en el glicol rico y pobre son inferiores al límite establecido, lo cual indica que no hay degradación por corrosión generalizada en el sistema.

\section{Ensayos electroquímicos de polarización lineal}

Se realizó ensayos electroquímicos de corrosión en probetas de acero al carbono en medios de agua removida y muestras de TEG utilizando la técnica de Resistencia a la Polarización (LPR o Linear Polarization Resistance) y la curva de $\mathrm{Tafel}^{4}$ para determinar las velocidades de corrosión. Los resultados se muestran en la tabla 4.

Tabla 4. Resultados de las velocidades de corrosión obtenidos por las técnicas electroquímicas LPR y Tafel.

\begin{tabular}{llll} 
Muestra & ba & bc & vc (mpy) \\
\hline TEG sin uso & $743,5 \mathrm{e}-3$ & $224,4 \mathrm{e}-3$ & $62,87 \mathrm{e}-3$ \\
TEG pobre & $742,3 \mathrm{e}-3$ & $136,9 \mathrm{e}-3$ & $48,45 \mathrm{e}-3$ \\
TEG rico & $858,8 \mathrm{e}-3$ & $174,0 \mathrm{e}-3$ & $122,6 \mathrm{e}-3$ \\
TEG nuevo + agua & $3374,7 \mathrm{e}-3$ & $188,3 \mathrm{e}-3$ & $13,38 \mathrm{e}-3$ \\
Agua condensada de TEG & $322,1 \mathrm{e}-3$ & $372,0 \mathrm{e}-3$ & 4,496 \\
\hline
\end{tabular}

ba = Constante anódica de Tafel, bc = Constante catódica de Tafel $\mathrm{vc}=$ Velocidad de corrosión 
Se observa el comportamiento electroquímico en corrosión acuosa de los diferentes sistemas para las muestras de TEG. Los resultados evidencian unas tasas de corrosión despreciables para el caso de los glicoles nuevo, pobre y rico y una tasa de corrosión de 4 mpy (milésimas de pulgada por año que equivale a $0,10 \mathrm{~mm}$ por año) para el agua removida del proceso.

\section{Evaluación del inhibidor de corrosión con el agua del proceso}

Con el fin de evaluar el inhibidor de corrosión Coastal 1755- $\mathrm{C}^{5}$ utilizado en el sistema para neutralizar la acidez y controlar la corrosión, se realizó ensayos de corrosión con glicol nuevo y con el agua removida del sistema de deshidratación utilizando dosificaciones de 10,20 y 30 ppm; los resultados obtenidos se presentan en las tablas 5 y 6

Tabla 5. Resultados de las velocidades de corrosión encontradas en glicol nuevo.Tafel.

\begin{tabular}{llll}
\hline Dosificación del inhibidor & ba & bc & vc (mpy) \\
TEG sin uso + 10 ppm inhibidor & 431,2 e-3 & 202,1 e-3 & 41,57 e-3 \\
TEG sin uso + 20 ppm inhibidor & 444,0 e-3 & 156,1 e-3 & 48,45 e-3 \\
TEG sin uso + 30 ppm inhibidor & 3,03 e-1 & 244,4 e-3 & 1,44 e-3 \\
\hline ba = Constante anódica de Tafel, bc = Constante catódica de Tafel \\
vc = Velocidad de corrosión
\end{tabular}

Tabla 6. Resultados de las velocidades de corrosión encontradas en agua removida del TGE.

\begin{tabular}{|c|c|c|c|c|}
\hline Dosificación del inhibidor & ba & bc & vc (mpy) & $\begin{array}{l}\text { Eficiencia de } \\
\text { inhibición }\end{array}$ \\
\hline \multicolumn{5}{|l|}{ Agua condensada +10 ppm } \\
\hline inhibidor & 318.6 e-3 & $0.411 \mathrm{e}-3$ & 4.07 & 9.5 \\
\hline \multicolumn{5}{|l|}{ Agua condensada +20 ppm } \\
\hline inhibidor & 269.3 e-3 & $337.8 \mathrm{e}-3$ & 3.168 & 29.5 \\
\hline \multicolumn{5}{|l|}{ Agua condensada +30 ppm } \\
\hline inhibidor & $207.0 \mathrm{e}-1$ & $269.2 \mathrm{e}-3$ & 3.01 & 33.1 \\
\hline
\end{tabular}

Figura 3. Ensayos de corrosión usando dosificaciones del inhibidor a 10, 20 y 30ppm.

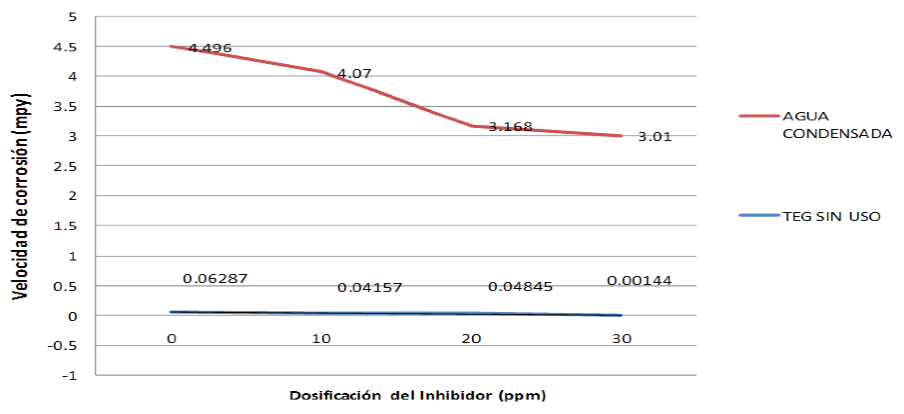


Los resultados de estos ensayos muestran que la velocidad de corrosión del agua de proceso sin inyección de inhibidor está en el orden de 4,5 mpy $(0,114 \mathrm{~mm} / \mathrm{año})$ y se reduce hasta un valor de $3 \mathrm{mpy}(0,076 \mathrm{~mm})$ con inyección de $30 \mathrm{ppm}$ en tanto que se registró valores despreciables de corrosión en el glicol en todos los casos (Fig. 3).

De los resultados de estos ensayos se deduce que con la inyección de inhibidor en el sistema las velocidades de corrosión no exceden de un valor de 4 mpy en el peor de los casos $(0,10$ $\mathrm{mm} / \mathrm{año}$ ).

La velocidad de corrosión es un parámetro que mide la pérdida de espesor de material por año, variable que no está normalizada sino que está en función del diseño del componente para el cual se considera una tolerancia de corrosión en función de la vida útil estimada. Por ejemplo, en un equipo cuya tolerancia de corrosión tenga por diseño un valor de $1 / 8 \mathrm{de}$ pulgada $(3,175 \mathrm{~mm})$, una tasa de corrosión de $4 \mathrm{mpy}$ resultaría en una vida útil que excede los 30 años por lo cual esta velocidad de corrosión se considera de baja magnitud ${ }^{6}$.

\section{Caracterización por TTIR del TEGN, TEGR y TEGP}

De los espectros obtenidos se puede corroborar que no se presenta degradación química del TEG rico y pobre con respecto al TEG nuevo (TEGN) ya que no se evidencia formación de compuestos de ácidos orgánicos como fórmicos o acéticos producto de la descomposición del trietilenglicol.

\section{CONCLUSIONES}

De las evaluaciones realizadas en las muestras de agua removida y del trietilenglicol se deduce que:

- El agua removida presenta parámetros de calidad aceptables para el servicio, considerándose un agua no corrosiva.

- La presencia del elemento azufre en el sistema de drenaje, como compuestos de sulfato de hierro en los depósitos, es un indicativo de contaminación del sistema aguas arriba en el proceso.

- Los valores reportados en los análisis para el TEG rico indican que la mezcla se encuentra entre los parámetros establecidos.

- No se evidenció compuestos de degradación del TEG rico y pobre.

- La velocidad de corrosión del agua de proceso sin inyección de inhibidor está en el orden de $4 \mathrm{mpy}(0,114 \mathrm{~mm} / \mathrm{Año})$ y para las mezclas de trietilenglicol se consideran despreciables; por lo tanto, el inhibidor Coastal 1755-C está neutralizando la acidez y controlando la corrosión en el sistema.

- Se recomienda usar un filtro inerte a los gases y líquidos.

\section{AGRADECIMIENTO}

Este estudio fue realizado con la coordinación del autor y de la empresa Brenntag distribuidora del aditivo Coastal 1755-C a la que agradecemos por su ayuda. 


\section{BIBLIOGRAFÍA}

1. Leyla Maritza Solano Guerrero. El gas natural en el Perú.Disponible en http://www. monografias.com/ trabajos58

2. Corredor, L., Hidalgo M. Informe Productec PPS-003-13-01. Noviembre 2013.

3. ASTM G-170- 01. Standard Guide for Evaluating and Qualifying Oil Field and Refinery Corrosion Inhibitors in the Laboratory.

4. Alvarado Cortés, J. Selección electroquímica de compuestos orgánicos como inhibidores de corrosión en medio ácido. [Tesis de grado]. Hidalgo. México: Universidad Autónoma del Estado de Hidalgo. 2006, p. 20-25.

5. Coastal 1755-C Product Information. From http://www.coastalchem.com

6. NACE Standard RP0775-2005. Preparation, Installation, Analysis, and Interpretation of Corrosion Coupons in Oilfield Operations. 